\title{
Urbanismo de mercado. Las ciudades latinoamericanas y el neoliberalismo realmente existente
}

\author{
Market urbanism. Latin American cities \\ and the neoliberalism that really exists
}

Ester Clelia Schiavo[l]
Alejandro Gabriel Gelfuso ${ }^{[I]}$

\begin{abstract}
Resumen
Si bien el neoliberalismo no se impone de forma homogénea, es posible englobar las distintas intervenciones bajo el concepto de urbanismo de mercado, motorizado por distintos modelos urbanos, principalmente por los denominados ciudad competitiva y ciudad inteligente. Ambos impulsados por organismos internacionales y empresas del sector privado, para luego ser adoptados por gobiernos locales a través de políticas públicas. Este proceso convive, no sin tensiones, con la consolidación de algunos gobiernos progresistas y la creciente apropiación normativa de conceptos como el derecho a la ciudad. El objetivo del presente trabajo es identificar y analizar las características actuales del neoliberalismo realmente existente en las ciudades latinoamericanas, para conocer los modos de implementación del urbanismo de mercado en la región.
\end{abstract}

Palabras clave: neoliberalización; gobiernos locales; modelos urbanos; sector financiero; movimientos sociales.

\begin{abstract}
Although neoliberalism is not imposed homogeneously, it is possible to include all the interventions in the concept of market urbanism, which is driven by different urban models, mainly by what is called competitive city and smart city. Both are promoted by international organizations and private sector companies, in order to be adopted by local governments through public policies. This process coexists, not without tensions, with the consolidation of some progressive governments and the increasing normative appropriation of concepts like "the right to the city". The objective of this paper is to identify and analyze the current characteristics of the neoliberalism that really exists in Latin American cities, to survey the forms of implementation of market urbanism in the region.
\end{abstract}

Keywords: neoliberalization; local governments; urban models; financial sector; social movements. 


\section{Introducción}

El neoliberalismo realmente existente se materializa en múltiples escalas geográficas y no se impone de forma pura ni homogénea, sino en función de contextos político institucionales moldeados por un legado de regulaciones, practicas institucionalizadas y hechos políticos transmitidos a través del tiempo (Theodore, Brenner y Peck, 2009). Por ende, se hace referencia a la existencia de diversos neoliberalismos, según sus contextos de inserción, y no a uno en particular. Sin embargo, es posible englobar estas múltiples intervenciones en el concepto de urbanismo de mercado que, en cada acción, reconstituye relaciones a distintas escalas entre los actores institucionales y económicos involucrados, como son los gobiernos locales y el sector financiero, particularmente articulado al inmobiliario, y simultáneamente, sustituye lógicas regulatorias por lógicas competitivas.

Dichas acciones se proponen mediante modelos urbanos paradigmáticos, impulsados por organismos internacionales y empresas del sector privado, para luego ser adoptados por gobiernos locales a través de políticas públicas. Los modelos de ciudad, arquetipos promovidos por distintos modos de asociación público-privada, se comportaron como hilo conductor para la restitución, por la vía institucional, de las políticas neoliberales implementadas a ultranza hasta finales del siglo XX, momento en el cual comienzan a transitar una relativa crisis de legitimidad.

Aun analizando casos concretos de ciudades donde persisten particularidades según el contexto de inserción, el objeto de estudio sigue siendo el mismo, la aplicación de políticas neoliberales; aunque cubiertas por diferentes ropajes, denominaciones y adjetivaciones. Ciudad competitiva, inteligente, sustentable, resiliente, participativa, inclusiva, creativa, innovadora, verde, educadora, entre otras; son algunas de las múltiples adjetivaciones que, con sus diferencias y matices, responden a un mismo patrón de transformaciones estructurales, similar a lo que algunos autores latinoamericanos denominaron la ciudad del pensamiento único (Arantes, Vainer y Maricato, 2000).

El objetivo del presente trabajo es identificar y analizar las características actuales del neoliberalismo realmente existente en las ciudades latinoamericanas, tanto como los principales ejes del debate y los modelos urbanos que se promueve imponer, con el propósito de conocer los modos de implementación del urbanismo de mercado en la región.

Una de las particularidades latinoamericanas en la etapa denominada por algunos autores como pos-neoliberalismo (Sader, 2008), radica en que el señalado proceso de neoliberalización de las ciudades mediante la promoción de modelos hegemónicos, en ciertos países ha convivido con la consolidación de gobiernos progresistas $y$, consecuentemente, con la apropiación normativa del derecho a la ciudad, que estos nuevos modelos estatales promueven.

En relación con lo señalado, una de las principales hipótesis que orienta este trabajo sostiene que, independientemente del contexto político aparentemente favorable al avance de determinados reclamos históricos de los movimientos sociales urbanos y la toma de 
compromisos por parte de distintos actores clave en dichos procesos, en la práctica, las ciudades latinoamericanas adoptaron preferentemente dos modelos hegemónicos de reproducción urbana. Los denominados ciudad competitiva y ciudad inteligente, más cercanos al urbanismo de mercado que a los postulados del Ilamado derecho a la ciudad (Lefebvre, 1968; Harvey, 2013). La elección de dichos modelos sobre el resto de los existentes, se explica por los intereses comunes del concierto de actores que los promueven e implementan, en relación con las transformaciones físicas y políticas de las ciudades del siglo XXI.

Esta disputa de modelos se reflejó, asimismo, en los debates suscitados en la Conferencia de las Naciones Unidas sobre la Vivienda y el Desarrollo Urbano Sostenible, conocida como Hábitat III y realizada en octubre de 2016 en la ciudad de Quito, Ecuador.

En función de la hipótesis planteada, algunos de los interrogantes que orientan el presente trabajo son los siguientes: ¿Cuáles son las características comunes del neoliberalismo realmente existente en las ciudades latinoamericanas? ¿Cuáles son las principales transformaciones urbanas en el continente a partir de la aplicación de políticas neoliberales durante tres décadas? ¿Cuánto tienen que ver estas políticas y sus postulados con la expansión de nuevos paradigmas como los de ciudad competitiva o inteligente? ¿Cómo se piensan, formulan y difunden las últimas agendas urbanas promovidas por organismos internacionales como el Banco Mundial (BM), el Banco Interamericano de Desarrollo (BID) 0 la Organización de las Naciones Unidas (ONU)?

A tal fin, se identifican y analizan las características del neoliberalismo realmente existente en las ciudades latinoamericanas, así como las complejidades y límites del posneoliberalismo. Perspectiva desde la cual se focaliza en el análisis crítico de dos de los modelos paradigmáticos dominantes, la ciudad competitiva y la ciudad inteligente; haciendo mención, a modo de referencia empírica, al caso argentino en general y a las ciudades de Rosario, Argentina, y Rio de Janeiro, Brasil, en particular. A posteriori, se consideran los tópicos analizados en el marco del debate suscitado en Hábitat III, tanto como la propuesta allí surgida de una nueva Agenda Urbana para las ciudades del mundo; para finalizar con el interrogante acerca del lugar que ocupa el urbanismo de mercado en esta mirada propositiva sobre las ciudades del futuro.

\section{Las ciudades latinoamericanas y el neoliberalismo realmente existente}

Las ciudades latinoamericanas aparecen como una combinación compleja y contradictoria de formas desigualmente desarrolladas, por ende, sólo es posible hacer generalizaciones sobre sus lógicas teóricas y los procesos que se expresan en la mayoría de los casos. Uno de ellos, el más estructural, es la eliminación del patrón de acumulación capitalista con intervención estatal, que permitió la industrialización y urbanización, entre otras cosas, y su reemplazo por el patrón neoliberal de acumulación, que trajo tres décadas signadas por fuertes recesiones, empleo informal creciente, pobreza y desigualdad. Características materializadas y concentradas en las ciudades, creando un 
modelo mundializado que, a pesar de no cumplir sus promesas originales, ha generado cambios sustanciales en la estructura, la gestión y el funcionamiento urbano, y que no solo no ha resuelto los problemas heredados, sino que ha generado otros nuevos y muy graves (Pradilla, 2013).

En las ciudades latinoamericanas, el programa concreto de materialización y reestructuración neoliberal combina, por un lado, el desmantelamiento de formas institucionales estatales que le son ajenas (como los sistemas redistribucionistas y colectivos) desregulando la economía al servicio de intereses corporativos; y por otra parte, promueve el lanzamiento de nuevas modalidades de regulación institucional y nuevas formas de gestión estatal. Lo que Theodore, Brenner y Peck (2009) denominan neoliberalización o neoliberalismo realmente existente.

Es este marco, la desindustrialización, proceso estructural que priva a las ciudades del sector más dinámico de la economía, cedió paso al resurgimiento del sector terciario, de servicios especializados, que carece de gran parte de las virtudes de la industria. En este devenir, la tercerización es acompañada por el fortalecimiento de un actor que aparece como sustituto de la industria en el espacio urbano: el sector inmobiliario, orgánicamente articulado al sector financiero, que se posiciona como un generador de valor y plusvalías mediante la renta del suelo. Es así que la expansión de la ciudad queda fuertemente condicionada por intereses privados, transformando a los territorios de las periferias metropolitanas, antes desvalorizados, en enormes reservas de tierra para grandes proyectos urbanos de diverso tipo. En tal sentido, la modernización urbana, la ciudad global, competitiva e inserta en un mercado local e internacional, se refleja en gran medida en la actividad inmobiliaria.

Otra transformación central de este proceso se cristaliza en el rol predominante de los gobiernos locales. En efecto, resultan actores relevantes, tanto por su potestad en lo que hace a la decisión sobre importantes variables económicas de la urbanización, como por sus significativas competencias en materia urbanística. Así, muchos de ellos, entre otras prácticas, a través de acciones u omisiones, impulsan legislaciones urbanísticas permisivas, favorecen urbanizaciones discontinuas, promueven grandes desarrollos urbanos y centralidades excluyentes o realizan recalificaciones de suelo en la periferia rural para favorecer a inversores privados, estimulando el llamado boom inmobiliario y las operaciones especulativas.

La globalización justifica y promueve la importación de políticas urbanas originadas en los países hegemónicos o en organismos multilaterales, impuestas mediante acuerdos comerciales, créditos y normas, muchas veces ajenas a los contextos de cada ciudad o región. La decisión de insertarse en ese nuevo orden global, compitiendo para posicionar a la ciudad como receptora de inversiones tiene efectos concretos en la conformación del espacio urbano.

Por una parte, el predominio del sector terciario en la estructura económica urbana, en su versión formal, tiende a crear nuevas centralidades mediante grandes centros comerciales, como los malls o los shopping centers que incluyen servicios bancarios, de esparcimiento y de gastronomía, entre otras 
actividades. Otras centralidades surgen en torno a zonas comerciales o de esparcimiento tradicionales, que se van fortaleciendo mediante la incorporación de múltiples actividades y de ese modo, adquieren una nueva identidad. Todos estos artefactos son un claro producto de las políticas neoliberales, orientados a sectores sociales con capacidad de pago individual, y se complementan, en las periferias urbanas, con barrios cerrados, cementerios privados y nuevamente, con grandes espacios donde predominan las actividades comerciales 0 de esparcimiento (Schiavo, 2004). Pero dichas políticas, como si se tratara de la otra cara de la misma moneda, paralelamente promueven distintas actividades informales, las que se despliegan bajo múltiples formas de tensión tanto en las áreas centrales como en las periferias urbanas, sometidas a la presión del sector inmobiliario financiero y profundizando de ese modo, la exclusión social y la fragmentación territorial (Schiavo, Vera y Dos Santos Nogueira, 2016).

Paralelamente, se asiste a un proceso tendiente a la privatización de las redes de infraestructura, los espacios y servicios públicos. Esto es, la transferencia parcial o total de las redes viales y los sistemas de transporte, los servicios de agua potable y desagües, la recolección de residuos y el saneamiento urbano en términos generales, los medios de comunicación y los espacios deportivos y recreativos. En suma, la significativa reducción del gasto público que deja un vacío ocupado por el capital privado, beneficiario privilegiado de este cambio de patrón que supone la construcción de obra pública y desarrollos privados.

\section{Complejidades y límites del pos-neoliberalismo}

Lo descripto anteriormente explica en parte por qué, si bien la región ha experimentado durante la última década un verdadero crecimiento en términos de inversión pública destinada a proyectos de infraestructura, en cuanto a la producción del espacio se refiere, la desigualdad social asociada a la fragmentación territorial sigue siendo la marca recurrente que acompaña a los procesos de crecimiento urbano. Se incluye, asimismo, en esta afirmación, a las experiencias políticas populares latinoamericanas que Ilegaron al poder a comienzos del siglo XXI y que dieron lugar a lo que se denomina Estados posneoliberales (Sader, 2008), en tanto poseen algunos rasgos comunes como el rechazo de la aplicación a ultranza de políticas de tinte neoliberal, la autonomía entre política y mercado, la nacionalización de algunos recursos estratégicos, el cuestionamiento a las formas institucionales tradicionales de la democracia liberal y el fomento del consumo interno, entre otras políticas y orientaciones comunes en la región. Se hace referencia, en particular, a países como Argentina, Bolivia, Brasil, Ecuador, Uruguay y Venezuela.

Así, en el marco de los cambios en el rol del Estado en su faceta pos-neoliberal, comienza a producirse una apropiación normativa del paradigma del derecho a la ciudad, que se inicia formalmente con la constituyente brasileña (1987-88), ${ }^{1}$ atraviesa una larga década con innovaciones y consolidaciones, como la instrumentación de 
la ley $388^{2}$ en Colombia sobre la recuperación de plusvalías urbanas, la redacción del estatuto de las ciudades en Brasil en $2001^{3}$ y la nueva constitución Ecuatoriana (y su código de ordenamiento territorial, COOTAD), ${ }^{4}$ para luego adquirir escala continental y mundial con la redacción de la carta internacional sobre el derecho a la ciudad, producida en Quito en $2004^{5}$ y luego, ratificada en Barcelona ${ }^{6}$ con el apoyo de Naciones Unidas y el Foro Urbano Mundial. Así, la promoción del derecho a la ciudad y con él, el impulso de políticas públicas urbanas para ciudades más justas, sustentables y democráticas se cimentaba en una alianza de principios entre gobiernos locales, agencias públicas nacionales y organismos internacionales. De este modo, a través de diferentes escalas de gobierno, se comienza a movilizar la noción del derecho a la ciudad como un marco conceptual lo suficientemente amplio como para contener, entre otros aspectos, una multiplicidad de iniciativas tributarias, sociales y de financiación de viviendas.

Desde otra perspectiva, se puede afirmar que las experiencias pos-neoliberales han mostrado preocupación por los avances de una urbanización excluyente, la precarización del hábitat y la desigualdad en el acceso a infraestructuras básicas. No obstante, y salvo pocas excepciones, no han adoptado políticas respecto a los territorios urbanos y al espacio residencial, dejando al suelo y la vivienda regidos por sus respectivos mercados. Siguiendo la metafórica mirada de José Natanson (2014) sobre el crecimiento en Latinoamérica, en dicho período, se puede decir que se presenta como un fenómeno de "puertas adentro", más que de "puertas afuera".
Un ejemplo visible es el mercado inmobiliario urbano, su lógica como bien de cambio y su influencia en los procesos de urbanización, que ha crecido notablemente en buena parte de las ciudades latinoamericanas, a pesar de la recuperación e instalación del discurso en favor de la soberanía pública estatal en áreas centrales de la economía (hidrocarburos, petróleo, agua, luz, aerolíneas, entre otros) y de los distintos compromisos asumidos por los Estados locales para construir ciudades más justas, como se expresó en Hábitat III.

En relación con lo señalado, otra de las hipótesis planteadas sostiene que el proceso de neoliberalización de las ciudades, es decir, la inserción contextual realmente existente del neoliberalismo se mostró inmutable a los cambios macro-políticos regionales. La discusión sobre la nacionalización o estatización de los bienes comunes, de gran consenso a favor de la recuperación de lo público, no solo no modifico la estructura ni la hoja de ruta del sector financiero-inmobiliario y el capital privado en general, de las grandes ciudades, sino que, paradójicamente, le dio más protagonismo gracias al crecimiento económico, profundizando la brecha entre quienes producen valor en la ciudad y quienes se apropian de los beneficios.

Así, en la actualidad, dichos modelos de ciudad evidencian claras anomalías y, en consecuencia, formas de acción colectiva y movilización que, por provenir de nuevos conflictos urbanos o de la profundización de los ya existentes, se presentan públicamente cuestionando el relato hegemónico de los modelos oficiales. Conflictos urbanos y demandas provenientes de sectores 
heterogéneos cuya característica común es que, ante el retroceso cuantitativo y cualitativo del Estado, deben resolver por sus propios medios sus necesidades básicas, particularmente las referidas al acceso a redes de infraestructura, equipamientos y servicios públicos. En general, teniendo frente a ellos al gobierno local solo como garante del statu quo de la propiedad urbana. Siendo, el mismo Estado el que manifiesta entre sus propósitos la idea de conducir un nuevo tipo de crecimiento sostenible con cohesión social.

Otra de las hipótesis que orienta este trabajo sostiene que, de la totalidad de acuerdos generales en torno a las agendas urbanas globales, en la práctica, los gobiernos locales sólo fortalecieron sus alianzas con organismos multitalerales, como el BID y el $B M$, con empresas del sector privado, como IBM, y con sectores inmobiliario-financieros, adoptando modelos paradigmáticos de construcción de ciudad que, la constante promoción de estos actores contribuyó a convertirlos en hegemónicos.

En consecuencia, es insoslayable la aparición de nuevas formas de organización, visibilización y acción que conforman los sujetos emergentes, desde los movimientos hasta las redes sociales y otras redes materiales heterogéneas, insertas en territorios acotados, pero no menos activos en sus reclamos por el derecho a la ciudad. La crisis de representación política que atraviesa en gran medida el continente latinoamericano también produjo el paso de luchas colectivas, de movimientos sociales urbanos, a agrupamientos y reclamos pluri-clasistas (lo que no significa que no existan determinaciones de clase) donde los sujetos sociales actúan en principio como individuos o ciudadanos que buscan nuevas formas de representación y resolución de sus conflictos cotidianos.

\section{Las ciudades competitivas}

Según el economista ecuatoriano Pablo Dávalos (2010), la transición conceptual que da nuevos fundamentos al neoliberalismo tiene fecha precisa: 1997, cuando el Banco Mundial publica su Informe de Desarrollo Mundial, ${ }^{7}$ centrado en la necesidad imperiosa de reconstruir el Estado, en un contexto de agotamiento y deslegitimación de las principales políticas económicas conducidas por los organismos internacionales de crédito.

El proceso de intervención sobre los marcos institucionales y sobre las sociedades de forma directa, por parte del Banco Mundial, ha sido denominado reforma estructural. De esta manera, mientras el Fondo Monetario Internacional (FMI) se concentraba en definir la hoja de ruta a través de los cambios en la macroeconomía, el Banco Mundial creaba el entramado institucional que le era correlativo, ayudado por la hegemonía teórica del neo-institucionalismo en la Ciencia Política. La ortodoxia y el fundamentalismo del FMI habían cerrado los espacios políticos a las transformaciones necesarias y habían movilizado a la sociedad en contra de los cambios institucionales previstos en la agenda neoliberal.

El cambio institucional propuesto por el Banco Mundial consiste en ajustes marginales al conjunto de reglas, normas y cumplimientos obligatorios que posibiliten la expansión del sector privado. La escala 
jerárquica privilegiada para estos cambios son las ciudades y sus respectivos gobiernos, aunque no necesariamente las metrópolis sino también otras localidades secundarias, de bajo perfil, pero con potencialidades. En ese marco territorial, el sector inmobiliario, orgánicamente articulado al sector financiero, aparece como el actor que sustituye a la industria en lo que hace a la generación localizada de valor, plusvalía y empleo (Pradilla, 2013)

Dichas reformas se enmarcan en un horizonte presentado bajo el nombre de Ciudad Competitiva, que se sustenta en un estudio sobre 750 ciudades en el período 20052012, estableciendo como diagnostico que el $72 \%$ de dichas localidades superó a sus países en cuanto a crecimiento económico. En función de lo cual sostiene que esta realidad debería profundizarse, si las ciudades aprovecharan sus ventajas comparativas para facilitar la generación de empleos, productividad e ingresos, implementando categorías de intervención entre las que se encuentran tres principales: instituciones y regulaciones, infraestructura y tierra y, finalmente, apoyo empresarial y financiero. ${ }^{8}$

El informe del Banco Mundial, como en otros momentos de la historia, se presenta como un conjunto de respuestas a una situación contextual que se considera difícil de administrar y exige coordinaciones inéditas.

Entre otras, se destacan dos respuestas para hacer competitiva a una ciudad. Por un lado, basándose en que la principal fuente de creación de empleos ha sido el crecimiento de empresas del sector privado, se sugiere que los Ilamados líderes de las ciudades deben familiarizarse con los factores que contribuyan a atraerlo y expandirlo. Por otra parte, se considera a las instituciones, las regulaciones y la infraestructura como los conductores determinantes de la competitividad. Paralelamente, como fenómeno económico relacionado con la capacidad de consumo, para el Banco Mundial la pobreza se convierte en una dimensión de la noción de crecimiento económico, cuyos ejes rectores están en el sector privado y en la lógica de los mercados autorregulados. Por ende, se estima que, para reducir la pobreza en las ciudades, el único camino considerado como plausible es aquel del crecimiento económico por la vía de estos mercados autorregulados (Dávalos, 2010).

En consecuencia, no se asiste a una simple retirada del Estado, sino a un nuevo compromiso político del Estado sobre nuevas bases, con nuevos métodos y nuevos objetivos. Principalmente, incorporando a los gobiernos locales como protagonistas de ese compromiso. Lo que ha pasado desapercibido es el carácter disciplinador de esta nueva política, que da al gobierno local el papel de guardián de reglas jurídicas, atribuyéndole la función oficial de controlador de las reglas de competencia en el marco de una coalición oficiosa con el sector privado, y asignándole el objetivo de crear o promover situaciones de mercado.

Esto habilita la reflexión sobre las consecuencias políticas de la aplicación del modelo de ciudad competitiva. Lo que Queiroz Ribeiro (2007) caracteriza como disociación entre urbs (la forma espacial y arquitectónica de la ciudad) y civitas (las relaciones humanas y políticas que se generan en ellas). Consideradas como dos dimensiones de la condición urbana, al unificarse representan el valor fundamental de las ciudades como invención social y, por el contrario, al 
disociarse se transforman en una anomalía política que se reproduce a escala intraurbana, abriendo grietas entre el progreso material, la urbanización, la economía y el territorio. Grietas que no pueden ser reconstruidas por el paradigma imperante de la competencia.

Para motorizar estos objetivos existen, según el citado informe del Banco Mundial, dos canales fundamentales. En primer lugar, las Ilamadas ventajas del alcalde: esto es, convertir el desarrollo económico en una prioridad explicita de los gobiernos locales. Y, en segundo lugar, la formación de coaliciones de crecimiento, que consisten en el fomento de las asociaciones público-privadas para la gestión de las políticas urbanas en todos sus niveles. No importa quién lleva a cabo las intervenciones clave, siempre y cuando alguien lo haga.

Por su parte, en general, los gobiernos locales encuentran que la actividad inmobiliaria, casi siempre muy visible, aporta legitimidad en el marco de las ideas en boga sobre la modernización urbana, la creación de iconos globales y la inserción en la globalización. Paradójicamente, esta integración de la economía mundial, lejos de reducir la importancia de las diferencias entre lugares ha venido a exacerbarla, de lo que se deriva que los territorios compitan entre sí a fin de atraer inversiones y puestos de trabajo; lo cual refuerza el paradigma de ciudad competitiva.

Entre otros países de América Latina, el Banco Mundial destaca como preocupación central el caso de las ciudades argentinas, cuyo historial de urbanización se remonta a la década del 30 del siglo pasado y donde habita el $91 \%$ de la población total del país (Naciones
Unidas, 2014). ${ }^{9}$ En Argentina, el desarrollo urbano se caracteriza por el desequilibrio en el contexto del desarrollo regional, la limitada presencia económica global de las economías urbanas y la expansión urbana de baja densidad no planificada. Según el informe, las ciudades argentinas enfrentan los desafíos de pasar de ciudades locales a ciudades globales y moverse de la expansión urbana de baja densidad a densidades articuladas para aprovechar plenamente los beneficios de las economías de aglomeración (Muzzini, Eraso Puig, Anapolsky, Lonnberg Mora, 2016).

Si bien los informes citados hacen hincapié en la identificación de economías en crecimiento y llama a potenciarlas, no se preguntan sobre la distribución de los beneficios del supuesto crecimiento, como tampoco profundizan sobre los modos de gestión que se aplicarían en torno a las economías ligadas a recursos naturales 0 bienes comunes urbanos, como la tierra por ejemplo.

Por otra parte, lo que parece estar en competencia no son las ciudades como un todo, sino los portadores de intereses económicos en ellas radicados. Las diferencias exacerbadas del lugar, en la práctica real del marketing local, tienden a reducirse a unos pocos rasgos a publicitar, minimizando sus complejidades y escondiendo contradicciones y conflictos.

\section{Las ciudades inteligentes}

La hegemonía de los modelos competitivos conlleva la emergencia de distintos tipos de adjetivaciones mediante las cuales se intenta promover la innovación en la gestión de 
las problemáticas urbanas. Promediando la segunda década del siglo XXI, las ciudades atraviesan momentos de crisis cuyos abordajes difieren según las características particulares de cada una de ellas. No obstante, como se señaló, se reconocen varios aspectos comunes en las ciudades latinoamericanas, entre otros, las transformaciones de las formas urbanas por diversos factores como la privatización de los servicios, la especulación inmobiliaria o la tercerización de la economía. Paralelamente, el rol de los gobiernos locales en la constitución de nuevos regímenes urbanos, se manifiesta acompañado por discursos sobre la ciudad que pretenden otorgar un sentido a sus acciones.

La expresión smart cities se acuñó hace más de una década y cuenta con un ranking propio en el cual se establece un listado de ciudades, ubicadas en distintas regiones del mundo, cuyas características coinciden con los criterios y definiciones hegemónicas de la inteligencia y su reflejo en las formas urbanas. Las smart cities o ciudades inteligentes, son el resultado de la necesidad cada vez más imperiosa de orientar políticas gubernamentales hacia la sostenibilidad. Así, las ciudades se sirven de infraestructuras, innovación y tecnología para disminuir el consumo energético y reducir las emisiones de $\mathrm{CO}_{2}{ }^{10}$

Durante la V Reunión sobre Ciudades Inteligentes y Sostenibles, realizada en junio de 2014 en Génova, Italia, se acordó la siguiente definición: "una ciudad inteligente y sostenible es una ciudad innovadora que aprovecha las tecnologías de la información y la comunicación y otros medios para mejorar la calidad de vida, la eficiencia del funcionamiento y los servicios urbanos y la competitividad, al tiempo que se asegura de que responde a las necesidades de las generaciones presente y futuras en lo que respecta a los aspectos económicos, sociales y medioambientales". ${ }^{11}$

El desarrollo tecnológico y la innovación se encuentran y extienden a prácticamente todos los ámbitos de la vida privada y pública, en parte gracias al impulso de grandes empresas como IBM. Es decir, se hace referencia a un paradigma cuyo actor principal es el capital privado y su cliente los Estados locales.

¿Cuáles son los parámetros que miden la inteligencia de una ciudad? En principio, se consideran 10 dimensiones de la vida urbana: gobernanza, planificación urbana, gestión pública, tecnología, medioambiente, proyección internacional, cohesión social, movilidad y transporte, capital humano y economía. El modelo en el que se sustenta el proceso de creación del indicador sintético es una agregación ponderada de indicadores parciales que representan cada una de las diez dimensiones que componen el modelo teórico. ${ }^{12}$

El estudio analiza 180 ciudades en 80 países (incluye 73 ciudades capitales), con base en 79 indicadores pertenecientes a las 10 dimensiones mencionadas. Los datos se obtienen de organismos y dependencias como la Unesco, el Banco Mundial y Euromonitor, aunque la disponibilidad de ciertos datos en determinados periodos se presenta como debilidad principal, debiendo extrapolar índices nacionales a ámbitos locales. Entre las citadas dimensiones se destacan la situación económica, el capital humano, el medioambiente, la tecnología, la movilidad y el transporte. Bajo este criterio, ciudades como Tokio, Nueva York, Londres, Paris, Zurich 
y Barcelona, entre otras, son consideradas entre las ciudades más inteligentes del mundo (Schiavo y Gelfuso, 2017a).

Como se dijo, es el sector privado a través de empresas multinacionales, quien promueve cambios radicales de paradigmas a nivel global que deben ser motorizados ya no por Estados nacionales sino a través de los gobiernos locales. Siguiendo a Dirks y Keeling (2009) del IBM Institute for Bussiness Value, se asiste a la adopción de un papel central de las ciudades en el mundo urbanizado del siglo XXI. Este nuevo orden contempla, en primer lugar, la caída de la producción como actividad económica, reemplazada por la prestación de servicios. En segundo lugar, el abandono del Estado nación y la aparición de un nuevo modelo de gobierno que ofrece más libertad a las ciudades en torno a la colaboración vertical entre diferentes niveles de la administración pública, así como mayores competencias legislativas y fiscales. El proceso de urbanización creciente implica que las ciudades tienen cada vez más control político y económico sobre su propio desarrollo, y por ende deben afrontar los retos y amenazas a su sostenibilidad.

Dentro de esta perspectiva de análisis, se concibe a la ciudad como la unión de 6 sistemas esenciales: personas, empresas, transporte, comunicación, agua y energía. Cada uno de ellos afronta retos y amenazas que, de no ser por un cambio de paradigma, afectarán la sostenibilidad de la vida urbana en las próximas décadas. Desde los cambios demográficos a la sanidad, los requisitos normativos, las cargas administrativas, los costos de movilidad, la demanda de conectividad, la eficacia en la distribución y calidad del agua hasta los sistemas energéticos inseguros e insostenibles.
Problemáticas que están interconectadas y deben afrontarse inmediatamente dotando de inteligencia a estos sistemas. Para ello, las administraciones locales han de decidir qué actividades son esenciales y cuales desechar, retener 0 ampliar.

Como consecuencia de este diagnóstico, las ciudades inteligentes se materializan e integran mediante elementos de infraestructura física y de servicios, como las tecnologías de iluminación, seguridad y energía renovable, utilizando la analítica inteligente. La industria tiene como objetivo generar cero emisiones y para lograrlo desarrolla técnicas de fabricación innovadoras. Las empresas de energía y de servicios públicos se sirven de redes inteligentes y comunicaciones inalámbricas; las empresas de gestión de aire, agua y desechos utilizan redes de sensores o sistemas de información; la seguridad se organiza a través de sistemas de video vigilancia. Los consultorios médicos ofrecen atención sanitaria a distancia y utilizan sistemas de gestión electrónica de los historiales médicos; las instalaciones educativas suministran contenido digital de la mejor calidad e imparten enseñanza interactiva y flexible. En definitiva, la sanidad, la educación, la movilidad o la agricultura: todo va a llevar adelante el adjetivo smart (Guillen, 2016).

La clave del paradigma, que también debe ser observada críticamente, es que gracias a la interconexión y monitorización de los sistemas esenciales de la ciudad se obtiene información valiosa, o sea: se producen datos. Más aun, la "inteligencia" consiste en la capacidad para utilizar la información generada, definir pautas de comportamiento o resultados probables y 
traducirlos en conocimiento real, permitiendo actuar de forma informada. Desde esta mirada, las ciudades inteligentes son uno de los principales campos en los que se materializará la explosión de conectividad que transformará la actual Internet de las personas, con 2.200 millones de internautas, en la Internet de las cosas, es decir, de objetos conectados. Se hace referencia a un debate que se presenta como central en el desarrollo urbano del siglo XXI (Schiavo y Gelfuso, 2017b).

Es evidente que, por su pertenencia a un organismo que promueve sus propios equipamientos y servicios dirigidos a gobiernos locales, las autoras del IBM Institute for Bussiness Value no profundizan ni problematizan críticamente algunos aspectos, tales como: ¿A quién pertenecen los datos producidos? ¿A los gobiernos locales, la ciudadanía en general 0 al capital privado que realiza parte de las inversiones y aporta el equipamiento? ¿Quién produce conocimiento en la ciudad inteligente y quiénes son sus beneficiarios reales? Estos interrogantes tienen un valor estimable en torno al futuro de las ciudades y definen un nuevo campo de disputa.

La simple interrogación sobre uno de los problemas históricos de las ciudades europeas y americanas, la pobreza y las condiciones indignas de vida (Peiró, 2015), pone en tensión algunos de los supuestos de este paradigma: ¿Puede autodenominarse inteligente una ciudad con graves problemas de tránsito y transporte público, con contaminación y con falta de transparencia en su gestión? Muchos sectores políticos de Latinoamérica y otros continentes afirman administrar ciudades inteligentes. ¿Con qué fines desarrollamos nuevas tecnologías? ¿Quién las construye, promueve y controla en última instancia? (Beas, 2014). ¿Qué tipo de desarrollo tecnológico se privilegia? ¿El de consumo dirigido a selectas minorías, centrado solo en la inyección de tecnología para estimular el control, o el que busca enfrentar grandes problemas sociales de largo plazo?

Para responder algunos de estos interrogantes, es interesante destacar la trayectoria de IBM en la última década, desde que comienza a impulsar su modelo de smart cities y a aplicarlo en distintas ciudades del mundo. A priori, se identifican dos propuestas originadas en el seno de la empresa. Una de ellas es la financiación, instalación y asesoría de gestión de Centros de Operaciones, cuyo objetivo es la creación de conocimientos analíticos en las operaciones con los gobiernos locales, a través de un punto central de comando. Estos centros toman distintas formas según la ciudad, aunque se destacan aquellos aspectos que hacen a la seguridad y el control. Un caso paradigmático es el de Rio de Janeiro, en Brasil, que desde 2010 cuenta con un centro de estas características, en el cual, además de la vigilancia, se recopilan datos de la actividad total de transporte, ambulancias y redes de agua ${ }^{13}$ para prevenir accidentes, inundaciones y problemas de tránsito, entre otros.

A partir de esta experiencia, desde 2011 IBM decidió expandir su zona de influencia hacia Argentina, también apostando a los Centros de Operaciones, ${ }^{14}$ pero fundamentalmente a partir del programa Smarter Cities Challenge, cuyo eje central es la capacitación de funcionarios en torno a la incorporación de la tecnología smart a la gestión de los gobiernos locales. Entre 100 ciudades preseleccionadas en el país, finalmente, la elegida fue Rosario, ${ }^{15}$ ciudad que 
también posee graves problemas ligados a la violencia urbana.

\section{Habitat III: el debate actual sobre las ciudades y los modelos en disputa}

Tal como se planteó hipotéticamente, los principales lineamientos de los dos modelos hegemónicos de reproducción urbana analizados en los apartados anteriores, también se implementaron en contextos políticos aparentemente adversos, como son ciertos países latinoamericanos que tuvieron o tienen gobiernos progresistas, donde se espera que prevalezcan posturas más próximas al derecho a la ciudad que al urbanismo de mercado. Esta tensión se refleja en los debates suscitados en la Conferencia de las Naciones Unidas sobre la Vivienda y el Desarrollo Urbano Sostenible, conocida como Hábitat III y realizada en 2016 en Quito, Ecuador.

Los distintos posicionamientos en dicha conferencia sirven para comprender la complejidad de la disputa en torno a los modelos hegemónicos adoptados por las ciudades latinoamericanas, y el contexto especifico en el que deben materializarse, allí donde los actores políticos, el sector privado y los movimientos sociales y ciudadanos conviven de manera conflictiva, entre acuerdos y desacuerdos, acciones y omisiones.

Hábitat III fue una conferencia sobre la vivienda y el desarrollo urbano sostenible que reunió a 193 Estados miembro de la Organización de las Naciones Unidas (ONU), con el objetivo de debatir temas urbanos y elaborar una agenda conjunta para que los organismos de los Estados, en sus diversos niveles, la tomen como guía para la acción gubernamental en torno a la construcción de ciudad.

Entonces, el producto final del encuentro, que se organiza cada 20 años, es la Nueva Agenda Urbana (2016) que se compone de 175 párrafos con tres principios fundamentales: no dejar a nadie atrás, economías urbanas sostenibles e inclusivas y sostenibilidad ambiental. Los ejes se refieren a medio ambiente, como la capacidad de adaptación de las ciudades frente al cambio climático, uso del agua, contaminación ambiental, manejo de desechos; universalización de los servicios públicos; y vivienda adecuada para la población. Se incluye también el concepto del derecho a la ciudad, entendida como un bien público, a la cual deben tener derecho todos los ciudadanos. Otros ejes de trabajo son el de género, el respeto a la diversidad, a las personas con mayores vulnerabilidades; seguridad, en el sentido de desastres naturales, protección de la vida; y desarrollo económico urbano. ${ }^{16}$

En lo que hace a la adopción concreta de medidas propuestas por la Agenda Urbana, el primer paso es que todos los niveles de gobierno definan estrategias de implementación de vivienda nacional o local basada en derechos humanos. Adicionalmente, que haya metas medibles sobre vivienda, protección a la población más vulnerable, mecanismos de revisión, monitoreo y solución de problemas. El segundo paso es el organismo evaluador de dichas medidas que, en el marco institucional del organismo mundial, está a cargo de la Asamblea Mundial de Gobiernos 
Locales y Regionales, ${ }^{17}$ acompañados por la secretaría general de la ONU.

No obstante, lo aparentemente amplia e inclusiva de la complejidad de la problemática formulada en la Agenda Urbana de Hábitat III, la conferencia contó con dos encuentros paralelos realizados en la misma ciudad, por actores no incluidos en el encuentro oficial.

Uno de los encuentros paralelos fue organizado por el Comité Popular por Nuestros Territorios, con el objetivo de promover la construcción participativa de la Agenda Autónoma Hábitat III desde la perspectiva de las necesidades de la comunidad, para difundir a la opinión pública los argumentos que permitan explicar los intereses que entrañan este tipo de conferencias mundiales y los impactos que tienen en el ambiente, en la economía, en la cultura y en la sociedad. También se propone poner en conocimiento los temas que denominan vedados, como son, entre otros, la violencia en barrios, el microtráfico, la inseguridad o la gentrificación. Es otras palabras, el principal cuestionamiento a la Declaración oficial de Quito es que encubre las causas y agentes responsables de los problemas y conflictos de las ciudades del siglo XXI.

La propuesta del Comité Popular culminó con la "Declaración por la defensa de nuestros territorios" ${ }^{18}$ documento elaborado por cientos de movimientos sociales locales e internacionales, cuyo postulado central es coordinar las luchas por la defensa de los territorios con un enfoque integral del hábitat, desarrollando acciones colectivas contra los desalojos y los despojos, y reivindicando el derecho a la ciudad, entendiendo que está compuesto por: derechos humanos, derecho a la tierra, al agua y a la vivienda, función social de la propiedad y producción social del hábitat. También se hace hincapié en la oportunidad de participar en verdaderos procesos democráticos en la toma de decisiones, desde la escala barrial a la escala nacional e internacional, advirtiendo sobre la clausura de espacios de discusión para la sociedad civil en el encuentro oficial.

Siguiendo otra de las hipótesis de este trabajo, la realidad actual de las ciudades latinoamericanas dista mucho de las propuestas oficiales, ya que el accionar de los gobiernos locales apunta mas a consolidar vínculos económicos con organismos multilaterales y empresas del sector privado antes que al cumplimiento de los compromisos tomados décadas atrás por esos mismos gobiernos. Sobre esta crítica se apoya el Manifiesto de Quito, ${ }^{19}$ producto de la otra reunión paralela a la conferencia Hábitat III, aprobado por los asistentes al Seminario Hábitat 3 Alternativo organizado por la Facultad Latinoamericana de Ciencias Sociales (Flacso), entre otros actores académicos. Al mismo asistieron las alcaldesas de Barcelona, Ada Colau, y de Madrid, Manuela Carmena, como también intelectuales y especialistas en urbanismo.

El Manifiesto de Quito afirma que la conferencia Hábitat III no se justifica, no se compromete y no representa. No se justifica porque las conferencias se realizan cada veinte años y los foros más frecuentes han permitido encuentros carentes de espacio de debate, resoluciones y seguimiento de los compromisos, como tampoco han tenido efectos visibles ni resultados prácticos en las ciudades. Por otro lado, la Agenda Urbana declara grandes compromisos, pero no responde a interrogantes como: ¿Quién los asume? ¿Los gobiernos 
nacionales? ¿El Programa de Naciones Unidas para el Desarrollo (PNUD) y Hábitat? ¿Los gobiernos de las ciudades? ¿Se concretan los compromisos en acciones o controles por parte de los gobiernos competentes? ¿Cuáles medidas se tomarían si se incumplen los compromisos? ¿Se podrá recurrir a tribunales internacionales? Por último, se pone en duda la representatividad de la conferencia al reducir la participación y la promoción del derecho a la ciudad a Estados y gobiernos.

Dicho Manifiesto sugiere que se cree una agencia independiente formada por destacados activistas sociales, expertos reconocidos y representantes o ex gobernantes de ciudades, que coordine una red de colaboradores que adviertan sobre el cumplimiento de los compromisos adquiridos. Las asociaciones de ciudades, las organizaciones sociales o cívicas y los centros de estudios e investigaciones podrían ser importantes colaboradores. Se trata, en definitiva, de reconstruir un Hábitat que no esté en manos exclusivamente de los gobiernos y los Estados.

En suma, tanto Hábitat III como los respectivos encuentros paralelos que la cuestionan, son la expresión del complejo debate en torno a modelos urbanos antagónicos, los promovidos desde el paradigma que privilegia el derecho a la ciudad frente a aquellos que impulsan el urbanismo de mercado.

\section{Reflexiones finales}

El urbanismo de mercado es la forma de reproducción dominante de las ciudades latinoamericanas. El análisis de dos de los modelos urbanos paradigmáticos, con los que se promueve e implementa dicha forma de desarrollo urbano, permitió identificar ciertas similitudes entre ellos, dado que ambos se basan en un mismo patrón de transformaciones estructurales.

Las similitudes entre el modelo de ciudad competitiva y el de ciudad inteligente, se encuentran en línea con el planteo hipotético que orientó este trabajo. En efecto, se verifica que, a pesar de la existencia de contextos aparentemente favorables al posneoliberalismo en ciertos países de la región, igualmente, la mayor parte de las ciudades optaron por seguir los lineamientos de los mencionados modelos hegemónicos, lo que inevitablemente conduce a privilegiar el urbanismo de mercado sobre la postura antagónica liderada por el derecho a la ciudad. Incluso, se puede afirmar que el neoliberalismo resultó inmutable a los cambios positivos generados en dichos países por los gobiernos progresistas dado que, por caso, el crecimiento económico benefició a uno de sus actores privilegiados, el sector inmobiliario-financiero.

En este marco, como también se planteó hipotéticamente, los gobiernos locales con los únicos que fortalecieron sus alianzas fueron con los actores estratégicos del urbanismo de mercado, es decir, los organismos internacionales y el sector privado. Aspecto asimismo destacado en los documentos de las reuniones alternativas de la conferencia Hábitat III.

En otras palabras, los modelos de ciudad hegemónicos, arquetipos aceptados por actores estratégicos del sector privado y por los gobiernos locales, se posicionaron como articuladores de intereses públicoprivados, cuyo poder de decisión en torno a 
la planificación urbana resulta central para fomentar un tipo de crecimiento excluyente. Por tal motivo, incluso en los gobiernos posneoliberales persiste la desigualdad social asociada a la fragmentación territorial. Lo que se caracterizó como crecimiento "puertas adentro" pero no "puertas afuera", dado que si bien en ciertos países la población aumentó sus niveles de consumo individual, no logró acceder en igual medida a los bienes comunes y a los equipamientos y servicios públicos.

No es de extrañar, entonces, el clima de conflictividad social y crisis de representatividad que se vive en buena parte de la región. Ambiente en el cual emergen sujetos sociales que visibilizan la falta de participación ciudadana en la elaboración de políticas urbanas y de gestión de las ciudades, que siguen siendo muy precarias $y$, por ende, con agendas sesgadas que no contemplan los problemas realmente existentes. Cuestiones en las que también se abundó en los documentos de las reuniones alternativas a la conferencia Hábitat III. Particularmente, en la Declaración por la defensa de nuestros territorios, resultante del encuentro promovido por múltiples organizaciones de la sociedad civil, se plantea explícitamente que la Declaración oficial de Quito encubre las causas y agentes responsables de los problemas y conflictos de las ciudades del siglo XXI.

En torno al modelo de ciudad inteligente surgen algunos interrogantes en particular, desde la mirada dominante todo el problema parece estar centrado en los datos, en la información, en cómo se produce y administra y en quiénes son sus dueños. Pero, teniendo en cuenta la precaria situación de millones de personas que viven en las ciudades del mundo, surgen otros interrogantes que relacionan a las ciudades inteligentes con la posibilidad de morigerar la pobreza y mejorar las condiciones de vida de los habitantes urbanos; lo cual plantea un nuevo campo de disputa.

Si asistimos a un modelo integrado, a un fenómeno global que atraviesa a las principales urbes del mundo y su principal objetivo es la sostenibilidad y la gobernanza ¿No es necesario anteponer a la ciudad inteligente la pregunta por el derecho a la ciudad? Si la respuesta fuera afirmativa ¿la materialización de una ciudad inteligente no debería partir de los interrogantes principales que hacen a la consolidación de ese derecho a la ciudad? El sentido de estos interrogantes también está presente en los documentos de los encuentros alternativos a la conferencia Hábitat III.

En cambio, la Nueva Agenda Urbana (NAU) acordada por los actores estatales en la conferencia oficial Hábitat III, parece separar los procesos urbanos de los modelos de ciudad hegemónicos que los promueven y de sus actores dominantes, sin reconocer responsabilidades en torno al accionar de estos últimos en dichos procesos. Asimismo, en la NAU, se observa otra contradicción: la incorporación del concepto de derecho a la ciudad, aunque reducido al acceso a bienes y servicios, promoviendo de ese modo procesos de tercerización de la economía urbana.

Tanto en la idea de ciudad inteligente o ciudad competitiva como en la de derecho a la ciudad, se dirime una disputa por su significación que, lejos de quedar limitada a un mero arreglo y juego discursivo, se despliega en formas opuestas de entender la participación, 
la producción del espacio urbano y la capacidad para experimentar arreglos socio-materiales más justos para habitar la ciudad. En el caso de las ciudades latinoamericanas, este aspecto es más profundo aun, debiendo atender las consecuencias sociales y físicas de procesos históricos de urbanización desiguales, signados por dinámicas espaciales expulsivas, donde las propias modalidades de acumulación urbana llevan a gobiernos locales a habilitar - y en muchos casos a propiciar la diseminación de una serie de intervenciones urbanas como tipologías exclusivas y grandes proyectos, que lejos de contrarrestar procesos de injusticia espacial, tienden a multiplicarlos y acentuarlos.

Las posturas dominantes confluyen en una mirada propositiva sobre las ciudades del futuro, en la que se profundiza la brecha entre quienes toman las decisiones fundamentales en torno a los procesos de urbanización y la adopción de modelos competitivos, en línea con el urbanismo de mercado, y quienes sufren las consecuencias de estas decisiones.

[I] http://orcid.org/0000-0003-4261-3756

Universidad Nacional de Quilmes, Departamento de Ciencias Sociales. Bernal, Buenos Aires, Argentina.

eschiavo@gmail.com

[II] http://orcid.org/0000-0001-5111-4727

Universidad Nacional de Quilmes, Departamento de Ciencias Sociales. Bernal, Buenos Aires, Argentina.

alegelfuso@gmail.com

\section{Notas}

(1) República Federativa de Brasil: Constitución de 1988. Disponible en: http://pdba.georgetown. edu/Constitutions/Brazil/esp88.html

(2) Ley Orgánica de Ordenamiento Territorial de Colombia. Disponible en: http://www.umng.edu.co/ documents/10162/745281/V3N2_24.pdf

(3) El estatuto de la ciudad: nuevas herramientas para garantizar el derecho a la ciudad en Brasil. Disponible en: http://www.cafedelasciudades.com.ar/imagenes/Estatuto\%20de\%20la\%20 Ciudad_Brasil.pdf

(4) Código Orgánico de Organización Territorial, Autonomía y Descentralización, Ecuador. Disponible en: http://www.ame.gob.ec/ame/pdf/cootad_2012.pdf. 
(5) Carta Mundial por el Derecho a la Ciudad. Disponible en: http://www.ugr.es/ revpaz/ documentacion/rpc_n5_2012_doc1.pdf.

(6) http://www.onuhabitat.org/index.php?option=com_docman\&task=doc_ details\&gid=50\&Itemid=3

(7) Banco Mundial (1997). Informe sobre el desarrollo mundial 1997: el estado en un mundo en transformación. Suiza, Work Bank Group. Disponible en: www.worldbank.org

(8) Banco Mundial (2015). Ciudades competitivas para el empleo y el crecimiento. Suiza, Work Bank Group. Disponible en: www.worldbank.org/competitivecities

(9) Programa de las Naciones Unidas para el Desarrollo-PNUD (2014). Informe sobre desarrollo humano 2014. Sostener el progreso humano: reducir vulnerabilidades y construir resiliencia. Disponible en: http://hdr.undp.org/sites/default/files/hdr14-summary-es.pdf.

(10) Sostenibilidad para todos. ¿Qué es una Smart city? Top 5 de ciudades inteligentes. Disponible en: http://www.sostenibilidad.com/que-es-una-smartcity-top-5-ciudades-inteligentes

(11) Grupo Tematico sobre ciudades sostenibles e inteligentes (2015). Disponible en: http://www.itu. int/es/ITU-T/focusgroups/ssc/Pages/default.aspx

(12) Centro de Globalización y Estrategia (2015) Índice IESE cities in motion. Navarra, Instituto de Estudios Superiores de la Empresa (IESE). Disponible en: http://www.iese.edu/research/pdfs/ ST-0366.pdf

(13) Disponible en: https://elpais.com/tecnologia/2012/03/16/actualidad/1331890089_208324.html.

(14) Disponible en: http://www.iprofesional.com/notas/120369-IBM-trae-a-la-Argentina-un-centropara-controlar-la-vida-de-las-mega-ciudades.

(15) Rosario es una ciudad de escala metropolitana, constituye el tercer núcleo urbano más poblado de Argentina. Se ubica en la zona sur de la provincia de Santa Fe, no siendo su ciudad capital, y tiene una posición estratégica en la región Mercosur gracias a su actividad portuaria en un contexto territorial conducido por los agronegocios. Posee 948.312 habitantes, a los que se suma una conurbación territorial que involucra aproximadamente 23 localidades, concentrando más de 1.400.000 ciudadanos (Censo 2010). Disponible en: https://www.lacapital.com.ar/laciudad/rosario-fue-elegida-ibm-desarrollar-el-programa-ciudades-inteligentes-n383028.html

(16) Conferencia de las Naciones Unidas para la vivienda y el desarrollo urbano sostenible (2016). Documento final Hábitat III. Quito. Disponible en: https://www2.habitat3.org/. che/907f3c56d3ad27a3daeeb677c660545a00c69d6b?vid=591158\&disposition=inline\&op=view

(17) Red Mundial de Gobiernos Locales y Regionales (UCLG). Información disponible en: https://www. uclg.org/es/eventos/quito-2016-2a-asamblea-mundial.

(18) Comité Popular en defensa de nuestros territorios (2016). Declaración final sobre la defensa de nuestros territorios. Resiste Hábitat III. Quito. Disponible en: https://resistenciapopularhabitat3. $\operatorname{org} / 2016 / 10 / 25 /$ declaracion-por-la-defensa-de-nuestros-territorios/.

(19) Facultad Latinoamericana de Ciencias Sociales - Flacso (2016). Manifiesto de Quito: hacia un Habitat III Alternativo. Quito, Flacso. Disponible en: https://www.flacso.edu.ec/habitat/. 


\section{Referencias}

ARANTES, O; VAINER, C. e MARICATO, E. (2000). A cidade do pensamento único. Desmanchando consensos. Rio de Janeiro, Vozes.

BEAS, D. (2014). Politizar la tecnología. España, El País digital. Disponible en: http://elpais.com/ elpais/2014/09/24/opinion/1411584405_269344.html. Acceso en: 10 maio 2018.

DÁVALOS, P. (2010). La democracia disciplinaria: el proyecto posneoliberal para América Latina. Ecuador, Codeu.

DIRKS, S. y KEELING, M. (2009). Ciudades más inteligentes, hacia un nuevo modelo de eficiencia y sostenibilidad. Dublin, IBM Institute for Bussiness Value.

GUILLEN, B. (2016). Elsueñodecrearelprimerpaísinteligente delmundo. España, El Pais digital. Disponible en: http://tecnologia.elpais.com/tecnologia/2016/05/23/actualidad/1463996571_677736. html?id_externo_rsoc=FB_CC. Acceso en: 10 maio 2018.

HARVEY, D. (2013). Ciudades rebeldes. Del derecho a la ciudad a la revolución urbana. Madrid, Akal.

LEFEBVRE, H. (1968). El derecho a la ciudad. Barcelona, Península.

MUZZINI, E; ERASO PUIG, B.; ANAPOLSKY, S.; LONNBERG, T. y MORA, V. (2016). Liberando el potencial de las ciudades argentinas. Marco de acción para las políticas públicas. Grupo Banco Mundial. Disponible en: http://documentos.bancomundial.org/curated/es/423671476947700973/ pdf/109359-PUB-PUBLIC-PUBDATE-10-18-16.pdf. Acceso en: 10 maio 2018.

NATANSON, J. (2014). Nuestro ogro filantrópico. Buenos Aires, Le Monde Diplomatique.

PEIRÓ, K. (2015). Barcelona ¿Smart City? Barcelona, NacioDigital. Disponible en: http://www. naciodigital.cat/opinio/11977/barcelona/smart/city. Acceso en: 10 maio 2018.

PRADILLA COBOS, E. (2013). "Empresarios, gobiernos y ciudadanos en la disputa por la ciudad". En: RIBEIRO, A. C. T. et al. Politica governamental e ação social no espaço. Río de Janeiro, Anpur.

QUEIROZ RIBEIRO, L. C. (2007). Metropolis brasileñas: ¿Cómo gobernar las urbs sin cívitas? Nueva Sociedad, n. 212.

SADER, E. (2008). Refundar el Estado. Posneoliberalismo en América Latina. Buenos Aires, Argentina, Ediciones CTA - Consejo Latinoamericano de Ciencias Sociales.

SCHIAVO, E. (2004). Des réseaux techniques urbains aux technologies de la société de l'information. Francia, Université de Lille, Atelier National de Reproduction de Thèses.

SCHIAVO, E.; VERA, P. y DOS SANTOS NOGUEIRA, C. (2016). La Salada: imaginarios y representaciones de la informalidad y las desigualdades territoriales en la prensa escrita. Revista Question. Universidad Nacional de La Plata, v. 1, n. 50. Disponible en: http://perio.unlp.edu.ar/ojs/index. php/question/article/view/3203/2744. Acceso en: 10 maio 2018.

SCHIAVO, E. y GELFUSO, A. (2017a). Ciudades inteligentes: ¿Cómo pensar indicadores focalizados en la sociedad y el desarrollo de Iberoamérica? En: X CONGRESO IBEROAMERICANO DE INDICADORES DE CIENCIA Y TECNOLOGÍA. Diálogo entre productores y usuarios de información. San José de Costa Rica. Disponible en: https://drive.google.com/file/d/1UbSzkEOMFOCHAuxfSdbziMsLelqT9 vKF/view. Acceso en: 10 maio 2018. 
SCHIAVO, E. y GELFUSO, A. (2017b). “Ciudades inteligentes y laboratorios ciudadanos: Adjetivando el derecho a la ciudad”. En: HIERNAUX, D. y GONZALEZ GÓMEZ, I. (coords.). La ciudad latinoamericana a debate: Perspectivas teóricas. México, Universidad Autónoma de Querétaro. Disponible en: file://C:/Users/Ester/Downloads/La\%20Ciudad\%20Latinoamericana\%20a\%20 Debate.\%20Perspectivas\%20Teo\%CC\%81ricas\%20VVVFFF\%20(2).pdf. Acceso en: 10 maio 2018.

THEODORE, N.; BRENNER, N. y PECK, J. (2009). Urbanismo neoliberal: la ciudad y el imperio de los mercados. Temas Sociales. Santiago de Chile, n. 66.

Texto recebido em 10/dez/2016

Texto aprovado em 17/abr/2017 